\title{
Corrigendum
}

\section{Corrigendum to "Automatic Segmentation and Enhancement of Pavement Cracks Based on 3D Pavement Images"}

\author{
Baoxian Li $\mathbb{D}^{1},{ }^{1}$ Kelvin C. P. Wang, ${ }^{1,2}$ Allen Zhang $\mathbb{D}^{2},{ }^{2}$ Yue Fei, ${ }^{2}$ and Giuseppe Sollazzo ${ }^{3}{ }^{3}$ \\ ${ }^{1}$ School of Civil and Environmental Engineering, Southwest Jiaotong University, Chengdu 610031, China \\ ${ }^{2}$ School of Civil and Environmental Engineering, Oklahoma State University, Stillwater, OK 74078, USA \\ ${ }^{3}$ Department of Engineering, University of Messina, Vill. S. Agata, C.da di Dio, 98166 Messina, Italy \\ Correspondence should be addressed to Allen Zhang; allen.zhang@okstate.edu
}

Received 28 April 2020; Accepted 13 May 2020; Published 11 September 2020

Copyright (c) 2020 Baoxian Li et al. This is an open access article distributed under the Creative Commons Attribution License, which permits unrestricted use, distribution, and reproduction in any medium, provided the original work is properly cited.

In the article titled "Automatic Segmentation and Enhancement of Pavement Cracks Based on 3D Pavement Images" [1], the author Dr. Giuseppe Sollazzo was omitted from the authorship list in error. The authorship list is, therefore, being updated to add this author due to his contribution to the ideas and analysis of the study. The final authors' list is Baoxian Li, Kelvin C. P. Wang, Allen Zhang, Yue Fei, and Giuseppe Sollazzo.

\section{References}

[1] B. Li, K. C. P. Wang, A. Zhang, F. Yue, and G. Sollazzo, "Automatic segmentation and enhancement of pavement cracks based on 3D pavement images," Journal of Advanced Transportation, vol. 2019, Article ID 1813763, 9 pages, 2019. 